\title{
Neurotechnologies for Design Product in the Economy of Transformation
}

Francesca Bonetti

Neocogita

francesca.bonetti@neocogita.com

ORCID 0000-0001-9707-6236
Giorgio Casoni

Alma Mater Studiorum

— Università di Bologna

giorgio.casoni@unibo.it

ORCID 0000-0003-4765-8275

\section{Abstract}

In an ever-changing world, it is essential to integrate different disciplines to create user-centred products and services. Design alone cannot fully understand the needs and requirements of the end users, nor the emotions and mental states they feel when they experience the product. There is a need for a multidisciplinary work that brings together the skills of designers with the most sophisticated neurotechnologies on the market. Neuroergonomics is an applied discipline that arises from the joint efforts of neuroscience and ergonomics. It uses existing knowledge about human behaviour and brain functioning to identify human-friendly technologies and interfaces and to support the design of safer and more efficient work environments that can increase worker well-being, while maximizing productivity. Thus, it can establish a reference framework for decision-making in the architectural and industrial design sector, focusing on central factors that affect people's reactions and behaviors.

\section{Keywords}

Neurotechnologies

Neuroergonomics

Transformation economy 
The rapid development of so-called "NBIC technologies" 1 is giving rise to possibilities that have long been a source of inspiration for science fiction novels and films (Roco \& Bainbridge, 2002).

Neurotechnologies are one of the concrete outcomes of this new technological wave. The term neurotechnology used here refers to devices/products designed "to investigate, evaluate, access and manipulate the structure and function of neural systems" (Giordano, 2012, p. 4) and, more generally, our nervous system. These can be neurons in the brain, neurons located in the spinal cord, cranial nerves, or any other type of sensory neurons.

These are devices destined for the final consumer and this effectively excludes all products/devices intended for medical applications. Consumer neurotechnologies (Bedows, 2018) are therefore devices designed to interact with neurons, not invasive, optimized and ergonomic so that they can be used by many users, that may or may not use other types of biological signals such as heart rate, heart rate variability, cortisol or eye tracking.

According to whatever the market offers, we can divide neurotechnologies in two main sets: the first set is composed of technologies used in medical and scientific research and practice, e.g., electroencephalography (EEG), electromyography (EMG) and transcranial direct current stimulation (tDCS); the second set concerns wearable sensors that may help understand our mental state and our inner world. The latter are wearable components (on the wrist, on the head or as glasses) incorporated in intelligent devices useful for monitoring several parameters such as blood pressure, movement, body temperature, heart rate, sleep, and muscle activity. Wearable sensors allow collecting, storing, and processing data to gain a better understanding of the user's health, in terms of both physical and mental status. In addition to producing information about the user, they can also detect information about the surrounding environment or about the user's interaction with the external world. The growth of the global wearable sensors market is mainly driven by advances in microelectronics, sensor manufacturing and telecommunications. The spread of wireless connectivity, combined with growing miniaturization, the decrease in costs and the integration with consumer electronic devices with Internet of Things (IoT), virtual reality and augmented reality platforms will increase the demand for wearable sensors in the coming years.

Neurotechnologies may represent a powerful tool for designers: the possibility of eliciting data on physiological, mental, and cognitive individual states represents a paradigm shift in design progress. It allows determining individual latent states that until recently were the prerogative of university research centers. Now designers can also access tools capable of representing mental and emotional states through which they can develop what we can define as data-driven design ${ }^{2}$.

The application of neurotechnologies finds its most complete use within the evolutionary process from the so-called Experience Economy to the Transformation Economy (Pine \& Gilmore, 2000). If the development of the Experience Economy characterized the approach followed by marketing almost two decades
Nano-Bio-Info-Cogno: nanotechnology, biotechnology, information technology and cognitive science

2

The authors intend a form of design that continuously considers the results of experiments carried out to test the e ectiveness of the product on the end user. 
ago, today the next evolutionary step is that of the Transformation Economy. In consumer processes, the brand, product, service, and purchase locations have been linked to the emotional sphere of the customer, giving rise to the so-called Experience Economy, retaining that experience constitutes a specific economic proposal. The idea of experience as a competitive lever is also introduced by those who believe that in order to differentiate goods it is no longer sufficient to increase their benefits, but it is important to offer the consumer an emotion. Therefore, experiences should "provide sensory, emotional, cognitive, behavioral and relational values" (Schmitt, 1999).

Today it is believed that the Experience Economy has run its course, giving way to the Transformation Economy where "the basis of success will lie in understanding the aspirations [emphasis added] of individual consumers and businesses and guiding them to fully achieve these aspirations" (Pine \& Gilmore, 2000, p. 214).

"The economic offer [in the Transformation Economy] is neither the materials it uses nor the physical goods it produces. Nor is it the processes it carries out or the meetings orchestrated by it. When a company leads the transformations, the offer is the individual" (Pine \& Gilmore, 2000, p. 214).

The concept of individual "aspiration" coincides with the so-called needs for self-realization which manifest themselves after all other needs (physiological, safety, social and esteem) have found satisfaction. According to Maslow (1964), the need for self-fulfillment represents the actualization of individual potential and ensures that the self-image coincides with the real situation. "A musician must play - claimed Maslow - a poet must write; a professor must teach; a man must be what he can be".

In the Transformation Economy, the emphasis on value creation shifts from its use to the user. From the phase in which experiences are described and staged, we progressively move to that in which the economic value is determined by guiding the transformations, thanks to an exact series of sequences of experiences best suited to guiding aspirants towards their goals (Pine \& Gilmore, 2000, p. 218).

According to Pine and Gilmore (2000, p. 219) "transformations ... cannot be extracted, produced, supplied, or even staged, but only guided. ... Nobody can force another person to change. All transformations take place in the client's very being, and therefore must be implemented by the client himself".

The use of neurotechnologies can be useful to analyze the lifestyle of the users to highlight gaps with respect to the goals to which they aspire, and to increase the interoceptive awareness of the functioning of their cognitive-emotional system, adopting training protocols to achieve the transformative goal.

In the following paragraphs we will try to understand why designers should exploit neurotechnologies to understand the end users' feelings and their emotional and cognitive states when using products. 


\section{Cognition in Design}

Identifying the cognitive and affective needs of a consumer is a complex and tricky challenge. The creation of products attributes that meet the consumers' personal wishes and needs in different contexts is a demanding defiance, because mismatches between the purpose of the companies and the consumer's goal may occur, indicating the need for products and services to become increasingly user-oriented.

In product design there are several cognitive-affective aspects to take into account (Tavares et al., 2021). When a product meets a consumer Fig. 1, it first encounters his senses (sight, hearing, touch, smell, taste). Subsequently it activates the affective system, the cognitive system, and past subjective experiences (memories, emotions, images, sounds, etc.). It elicits cognitive-affective responses and opinions in the consumer, which are then translated into behaviours. This output, coming from the consumer, should be considered for the development of new products or for the improvement of existing ones, in a perspective of a continuous product-user interaction.

It is our opinion that design and architecture should always consider the abovementioned model (Tavares et al., 2021) of interaction between product and consumer/user; listen to his feedback, suggestions, but also reactions and behaviours he is not always aware of. Usually, the opinion that the user expresses verbally when using a product is only the tip of the iceberg, hiding non-verbal behaviours that can give important information about the user-experience of the product/instrument/environment.

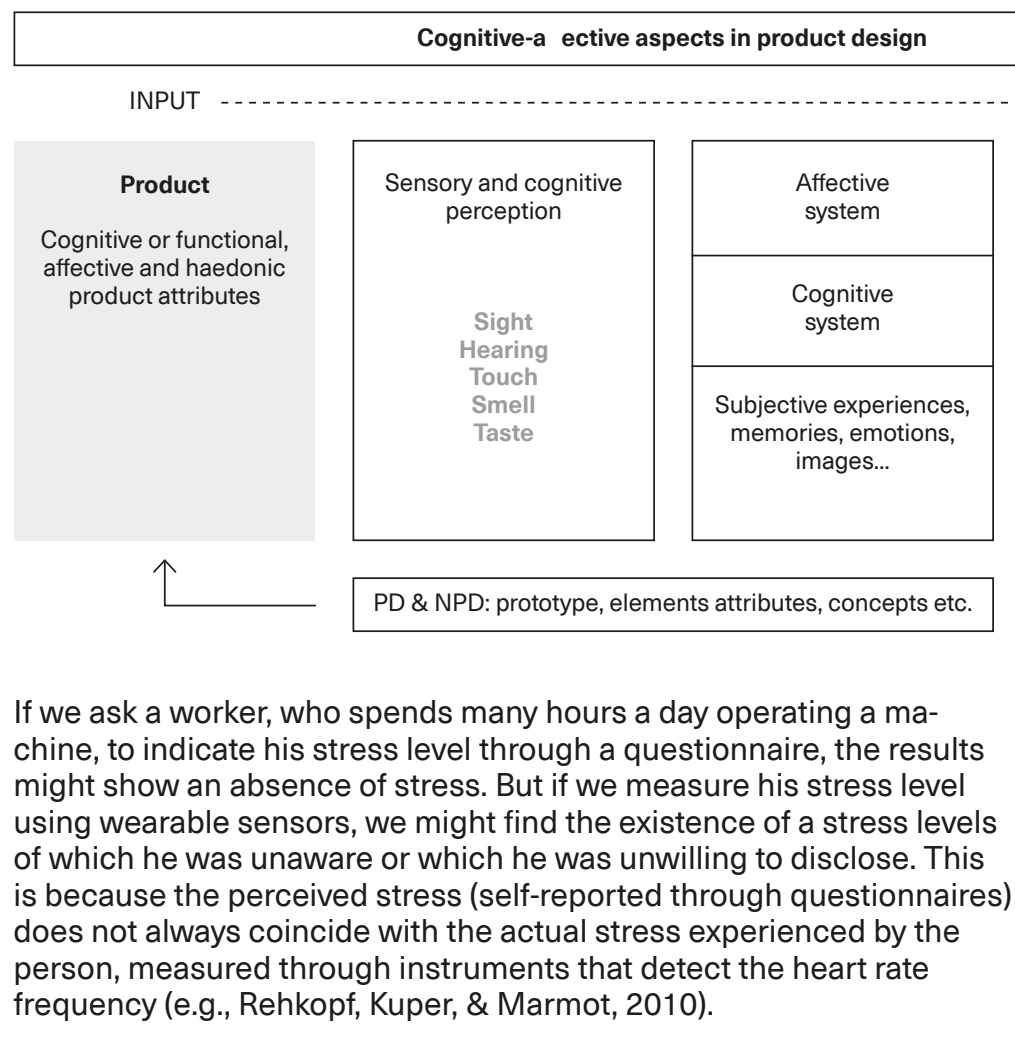


The need to create user-oriented products lies at the heart of neuroergonomics (Parasuraman, 2003), a discipline that arises from the fusion of neuroscience and ergonomics, namely between the scientific study of the brain to identify its structure and functionality (neuroscience), and the study of human-machine interaction in natural work and non-work contexts (ergonomics). Neuroergonomics uses existing knowledge about human behaviour and brain functioning to identify human-friendly technologies and interfaces, and to support the design of safer and more efficient work environments that can increase worker well-being while maximizing productivity.

Neuroergonomics should establish a reference framework for decision-making in the architectural and design sector (Causse et al., 2013), focusing on central factors that affect people's reactions and behaviors, and supporting the answer to questions like: how can we reduce stress, improve cognition, increase productivity in a workplace?

In this respect, neuroergonomics uses a range of tools taken from scientific research to answer questions pertaining to real-world scenarios. Some of the most used tools are listed below.

\section{Eye-Tracker}

It is a sophisticated tool that can record and analyse eye movements in order to identify the most salient areas of the visual field. It allows to obtain important metrics such as the amount of time the gaze spend on a given location, the frequency with which the gaze has stopped in a specific area, and the pupillary diameter, which can be used as an index of how busy or overloaded the individual's brain is at a given time. There are two measures that can be used to study the cognitive state of a person: pupil dilation and blink frequency.

Pupil dilation. Our pupils are constantly dilating and contracting. These changes in pupil diameter are mostly due to brightness, but they also occur when we experience particular emotions (e.g. fear, anger, joy, surprise) or when we are focused on a task. In this regard, it has been shown that pupil dilation, modulated by the locus coeruleus ${ }^{3}$ of the brain, reflects the so-called cognitive load, i.e. the mental effort required at a given time, when we are performing reasoning or reading tasks. Some studies have shown that the pupil tends to dilate when the cognitive load increases, and then decrease when this load exceeds the limit of our available cognitive resources (Granholm et al., 1996).

Blink frequency. The spontaneous blinking frequency correlates with dopamine levels in the central nervous system and may reveal the processes underlying learning and goal-directed behavior. Studies have shown that the number of blinks decreases as cognitive load increases (Benedetto et al., 2011; Ledger, 2013). This means that the more focused we are, the more we are using our cognitive resources up to the limit of availability, the less we blink. In working contexts, it is very important to highlight tasks is one of the re-organisations and sorting points for information that travels along ascending and descending pathways. 
that require excessive cognitive load and try to modify them. The reduction of cognitive load has an extremely positive effect on the number of errors made by the worker (Biondi et al., 2020; Tsai et al., 2007). It is therefore essential to identify the dysfunctional processes that prevent the worker from achieving maximum productivity, trying to remove all those elements that hinder the proper execution of a task.

\section{Electrocardiogram}

Electrocardiogram (ECG) data can be used to provide information about participant's physiological and emotional arousal in many fields and settings. The hands-free application combined with the high-fidelity data and wide-ranging analysis methods make the ECG well suited for understanding behaviour across both academic and industry research. One of the most informative metrics relies not just on the heart rate, but how much the heart rate varies, i.e. the Heart Rate Variability (HRV), an indirect measure of autonomic nervous system activity. In a healthy heart there is normal variation, in terms of milliseconds, between heartbeats. In a stressed heart, in contrast, the heartbeats are more constant, and this variation tends to be reduced. A low HRV value, therefore, is an indication of poor parasympathetic activity due to stress.

\section{Galvanic Skin Response}

Skin conductance or Galvanic Skin Response (GSR) originates from the autonomic activation of sweat glands in the skin, which reflects the intensity of the individual's emotional state. Our skin reveals a lot of information on how we feel when we are exposed to emotionally loaded images, videos, events, or other kind of stimuli - both positive and negative. No matter whether we are stressed, nervous, fearful, psyched up, stoked, baffled, or surprised - whenever we are emotionally aroused, the electrical conductivity of our skin subtly changes. The sweating on hands and feet is triggered by emotional stimulation: whenever we are emotionally aroused, GSR data shows distinctive patterns that are visible with bare eyes and that can be quantified statistically. With GSR, it is possible to tap into unconscious behaviour that is not under cognitive control. Skin conductance is modulated by autonomic sympathetic activity that drives bodily processes, cognitive and emotional states as well as cognition on an entirely subconscious level (Heard, 1964). We simply cannot exert conscious control over skin conductivity. This is precisely what makes GSR the perfect marker for emotional arousal, as it offers powerful insights into physiological and psychological processes of a person. 


\section{A Neuroergonomics Example: Smart+}

An example can help to better understand the development potential between neuroscience, neurotechnology and design (Casoni \& Celaschi, 2020). The concept of reference in this case is the "circadian rhythm". All biological organisms depend on the rhythms and oscillations of the activities that take place over time. When time is altered, our biological systems begin to fail. An example is the phenomenon of jet lag when we travel for trips to geographical areas with different time zones. In these cases, we notice the temporary difficulties of behavioral and physiological adaptation to the different time conditions compared to those of our departure. Far more serious are the consequences associated with shift works, where the most significant damage to the biological timing system is documented (Rajaratnam et al., 2013).

As mammals, the main regulator that controls circadian rhythms is found in the Suprachiasmatic Nuclei (SCN) in a brain region known as the hypothalamus. The SCN is a clock that provides circadian information to all cells in the body to adjust their physiology based on the time of day. The SCN maintains and regulates the rhythms of the physiological and behavioral systems 24 hours a day. Any disturbance of this circadian system caused externally by inappropriate lighting information or by other conflicting environmental signals, or internally, by a loss of SCN function or other neurological problems, causes damage to individual health and well-being. The lighting information sent to the SCN, which measures the time and intensity of the lighting, comes from a single sensory apparatus in the retina, separate from other photoreceptors such as cones and rods, which are used for standard vision (recognition of objects, color vision, motion detection, etc.).

This third photoreceptor is made up of cells that express a photo-pigment called melanopsin. Melanopsin is particularly sensitive to short light waves of the visible spectrum corresponding to blue. It communicates the information collected directly to the brain, through the SCN, which is responsible for regulating the circadian rhythm. This means that melanopsin is not responsible for the formation of the visual image, but is a simple blue light detector, fulfilling the function of switch/regulator of our circadian rhythm.

Considering the photobiological effects of light on the human body and its circadian significance (Wurtman, 1975; Pauley, 2004), the reception of light seems to be extremely important in the design of everyday products.

In this regard, in 2019 Neocogita, commissioned by a lighting company, supported a group of designers in the development of "SMART+", a dynamic light table lamp capable of improving the performance of activities that require concentration and attention. The luminous characteristics affect the different cognitive performances, and therefore it was decided to design a light in reference to a specific mental state rather than to the type of space to which it would be destined. The smart mode involves the use of a dedicated app, which contains a reference library explaining the scientific evidence behind the different functions available and accompanies a continuous flow of personal, physiological, and behavioral data, which are entered manually or through interfaces 
with other body monitoring apps. The lamp delivers a type of direct dynamic light that follows a programming of different light cycles preset in the intensity values, spectral distribution, and type of direct light distribution.

To validate this innovative product, an attention test was performed to compare the performance of two groups of participants subjected to two distinct types of lighting, but in a single spatial and temporal context. A letter-recognition task, which measures sustained attention, was submitted to the experimental group A using dynamic project lighting (provided with a prototype), and to the control group $B$, which instead used a traditional hanging light. The result confirmed expectations on experimental group $A$, which recorded a significantly better performance than control group $B$, and an improvement in the well-being perceived individually.

The product can interpret the light and time context through a time-of-flight distance sensor, which uses a small invisible light laser emitter to determine at what height the light source is positioned. The clock inside the lamp autonomously manages the alternation of execution of day and evening cycles relating to tasks of attention. A passive infrared sensor recognizes the micro-movements emitted by the user and identifies when the user leaves, tracks break and adjusts pause/exercise cycles. Different Fresnel ring optical schemes allow the uniformity of the diffused light and the closure of the light beam to a limited area.

As previous studies have shown (e.g., Biondi \& Rognoli, 2007; Ferraris, 2007; Hettinger et al., 2003), neuroscience and design are disciplines that, although different, should interact continuously in order to offer an effective product. According to Bridger (2017), this need for integration and interaction has given rise to neurodesign, a new discipline that applies insights from neuroscience and psychology to create new and more effective types of design. It exploits the way the brain processes visual stimuli to take decisions. The knowledge of the mechanisms of perception is crucial in this respect, because it makes it possible to exploit the main stages of human perception, i.e. colour perception, shape perception and meaning attribution. Although neurodesign is a developing discipline, it seems to be a growing trend among designers who put the needs and expectations of end users first.
Francesca Bonetti She has a master's degree in Psychology and a Ph.D. in Cognitive neuroscience. She works with Neocogita as a neuroscientist, and integrates the knowledge gained from academic research with the development of new human-centred products, services, and interfaces.

\section{Giorgio Casoni}

He was Professor of Economics and Innovation Management at the Università degli Studi di Urbino, the Politecnico di Torino and Milano. He teaches innovation management and neuroscience-driven innovation at Bologna Business School. He is CEO of Neocogita, a startup that develops technological systems and services that apply neuroscience for corporate well-being. 
Bedows, A. (2018). Consumer Neurotechnology: Volume 1.

Benedetto, S., Pedrotti, M., Minin, L., Baccino, T., Re, A., \& Montanari, R. (2011). Driver workload and eye blink duration. Transportation research part F: traffic psychology and behaviour, 14(3), 199-208. https://doi.org/10.1016/j. trf.2010.12.001

Biondi, F.N., Cacanindin, A., Douglas, C., \& Cort, J. (2020). Overloaded and at Work: Investigating the Effect of Cognitive Workload on Assembly Task Performance. Human Factors, 0018720820929928. https://doi.org/10.1177/ 0018720820929928

Biondi, E., \& Rognoli, V. (2007). Neuroscienze per il design. How neurosciences can help design. diid disegno industriale - industrial design, 27, 76-83.

Bridger, D. (2017). Neuro design: Neuromarketing insights to boost engagement and profitability. Kogan Page Publishers.

Casoni, G., \& Celaschi, F. (2020). Human Body Design: Corpo e progetto nell'economia della trasformatività. FrancoAngeli.

Causse, M., Dehais, F., Péran, P., Sabatini, U., \& Pastor, J. (2013). The effects of emotion on pilot decision-making: A neuroergonomic approach to aviation safety. Transportation research part C: emerging technologies, 33, 272-281. https://doi.org/10.1016/j. trc.2012.04.005
Ferraris, S. (2007). Transferring new knowledge from the field of neuroscience to product design education. In DS 43: Proceedings of E\&PDE 2007, the 9th International Conference on Engineering and Product Design Education, University of Northumbria, Newcastle, UK, 13.-14.09. 2007.

Giordano, J. (2012). Neurotechnology as Demiurgical Force: Avoiding lcarus' Folly. In J. Giordano (Ed.), Neurotechnology. Premises, Potential, and Problems. CRC Press, Taylor \& Francis Group. https://doi. org/10.1201/b11861

Granholm, E., Asarnow, R. F., Sarkin, A.J., \& Dykes, K.L. (1996). Pupillary responses index cognitive resource limitations. Psychophysiology, 33(4), 457-461. 10.1111/j.1469-8986.1996. tb01071.x

Heard, G. (1964). The psychogalvanic response in the study of sympathetic activity. British Journal of Surgery, 51(8), 629-631.

Hettinger, L.J., Branco, P., Encarnacao, L.M., \& Bonato, P. (2003). Neuroadaptive technologies: applying neuroergonomics to the design of advanced interfaces. Theoretical Issues in Ergonomics Science, 4(1-2), 220-237. https://doi.org/1 $0.1080 / 14639220210000$ 20918

Ledger, H. (2013). The effect cognitive load has on eye blinking. The Plymouth Student Scientist, 6(1), 206-223. http://hdl.handle. net/10026.1/14015
Maslow, A. (1964). Motivation and Personality. Harper and Row.

Parasuraman, R. (2003). Neuroergonomics: Research and practice. Theoretical issues in ergonomics science, 4(1-2), 5-20. https:// doi.org/10.1080/ 14639220210199753

Pauley, S.M. (2004). Lighting for the human circadian clock: recent research indicates that lighting has become a public health issue. Medical Hypotheses, 63(4):588-96. https://doi.org/10.1016/j. mehy.2004.03.020

Pine, B.J., \& Gilmore, J.H. (2000). L'economia delle esperienze. Etas. (Original work published: 1999, The Experience Economy: Work is Theatre and Every Business a Stage. HBS Press).

Rajaratnam, S.M., Howard, M.E., \& Grunstein, R.R. (2013). Sleep loss and circadian disruption in shift work: health burden and management. Medical Journal of Australia, 199, S11-S15.

Rehkopf, D., Kuper, H., \& Marmot, M. (2010). Discrepancy between objective and subjective measures of job stress and sickness absence. Scandinavian Journal of Work, Environment \& Health, 36(6), 449457. http://www.jstor.org/ stable/41151516
Roco, M.C., \& Bainbridge, W.S. (2002). Converging technologies for improving human performance: Integrating from the nanoscale. Journal of nanoparticle research, 4(4), 281-295. https://doi.org/10.1023/ A:1021152023349

Schmitt, B.H. (1999). Experiential Marketing. The Free Press.

Tavares, D.R., Junior, O.C., de Macedo Guimarães, L.B., \& Rudek, M. (2021) A Systematic Literature Review of Consumers' Cognitive-Affective Needs in Product Design From 1999 to 2019. Frontiers in Neuroergonomics, 1, 5. https://doi.org/10.3389/ fnrgo.2020.617799

Tsai, Y.F., Viirre, E., Strychacz, C., Chase, B., \& Jung, T.P. (2007). Task performance and eye activity: predicting behavior relating to cognitive workload. Aviation, space, and environmental medicine, 78(5), B176-B185.

Wurtman, R.J. (1975). The effects of light on the human body. Scientific American, 233(1), 69-77. 
